\title{
THE EFFECT OF DIFFERENT METHODS OF SELECTION ON THE FIXATION OF HYBRIDS.
}

\author{
By W. J. SPILlmaN, Washington, D. C.
}

Before we had knowledge of Mendelian principles it was supposed that any type could be fixed by continual selection to that type. This was partly true and partly not. If the type we select consists only of recessive characters we now know, that, in so far as Mendelian characters are concerned, it is fixed after the first selection. Furthermore, we know that if the type we are selecting is heterozygote, no amount of selection will fix it. The old rule was applicable only to selection of dominant types, as is seen in what follows.

Continued selection of the dominant type results in the gradual increase of that type, so that at the end of ten generations very nearly all the population will consist of the type selected. We will first consider the case of those plants that are self fertilized, or in which self fertilization can be accomplished artificially.

Let us take a cross between two varieties of wheat, one of which has velvet chaff and smooth heads and the other of which has glabrous chaff and bearded heads. In the second generation we get nine types, which differ in their gametic constitution but which are grouped into four classes which can be distigushed from external characters. If no selection is made in the second generation, all the seed, or an aliquot part of it, being planted the next year, and this is continued for ten generation, we get the results shown in Fig. 1. Here we see that the population gradually splits up into four fixed types, each constituting 25 per cent of the whole, with a very small percentage of hybrids between these types present. If we had been dealing with parents which differ in only one respect they would have split up into two types; if there had been three differences the number of types would have been eight, and so on, the general rule being, in the case of close fertilization, that the number of fixed types into which the descendants of the original hybrid. separate is 2 raised to a power indicated by the number of Mendelian character pairs in which the parents differ. Thus, if they differ in five respects the progeny of the hybrid will split up into 32 fixed types when there is no selection. With our present knowledge of Mendelian principles we could, in such a case, merely produce the hybrid, then sow its seed for several years, with no effort at selection, at the end of which 
time we could, by sélecting out individual plants and keeping their progeny separate, secure any of the fixed types present in the population.

Fig. 2 shows the results that follow in the same cross when all recessives that occur are discarded. That is, when all the plants which have velvet beards and smooth heads are saved; the others being destroyed.

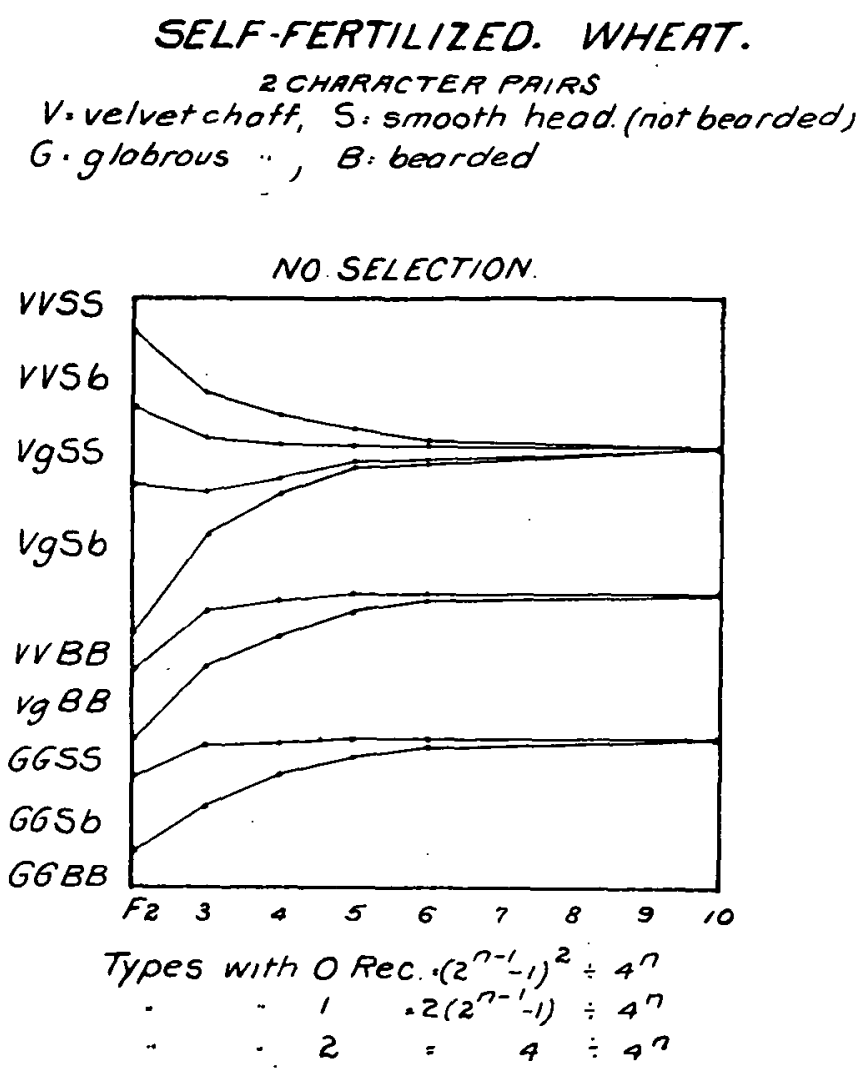

FIG. 1

In ten generations we see that our population becomes almost pure velvet chaff, smooth-headed wheat. This is what occurred under old methods of selection when the type selected happened to consist of dominant Mendelian characters.

But by taking advantage of Mendel's law in cases of this kind we can, by the third generation, secure in a fixed form the dominant type sought' 
The corresponding result is shown in Fig. 3. The method of accomplishing this is in the second generation to discard everything which does not have velvet chaff and smooth heads, the dominant characters. Save the seed of each plant separately, then in the next generation those

\section{SELF-FERT/LIZED. WHEAT.}

2 CHARACTEA PAIPS.

$V$-velvet chaff; $S$ : smooth head.(not beorded)

G.glabrous "; B-bearded.

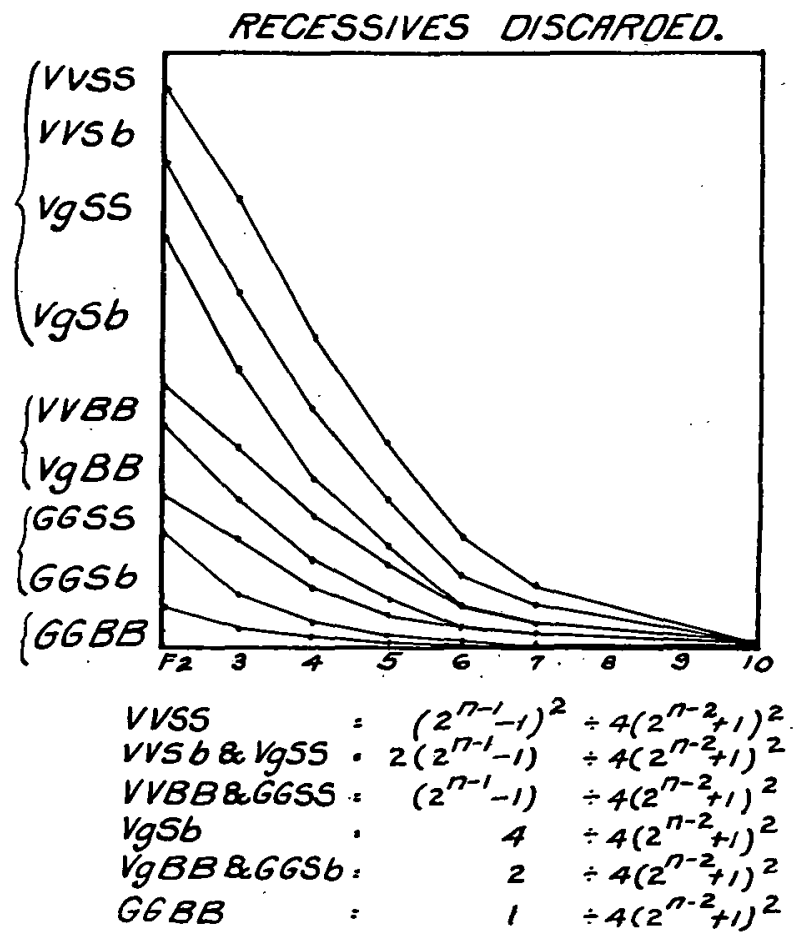

FIG. 2

plants which were homozygote in the preceding generation are discovered, and by propagating from them exclusively we have a fixed type.

In the case of animals and those plants which cross-fertilize the case is different. In the first place, when there is no selection there is no tendency for the population to split up into fixed types as there is when 
344 DIFFERENT METHODS OF SELECTION ON FIXATION OF HYBRIDS.
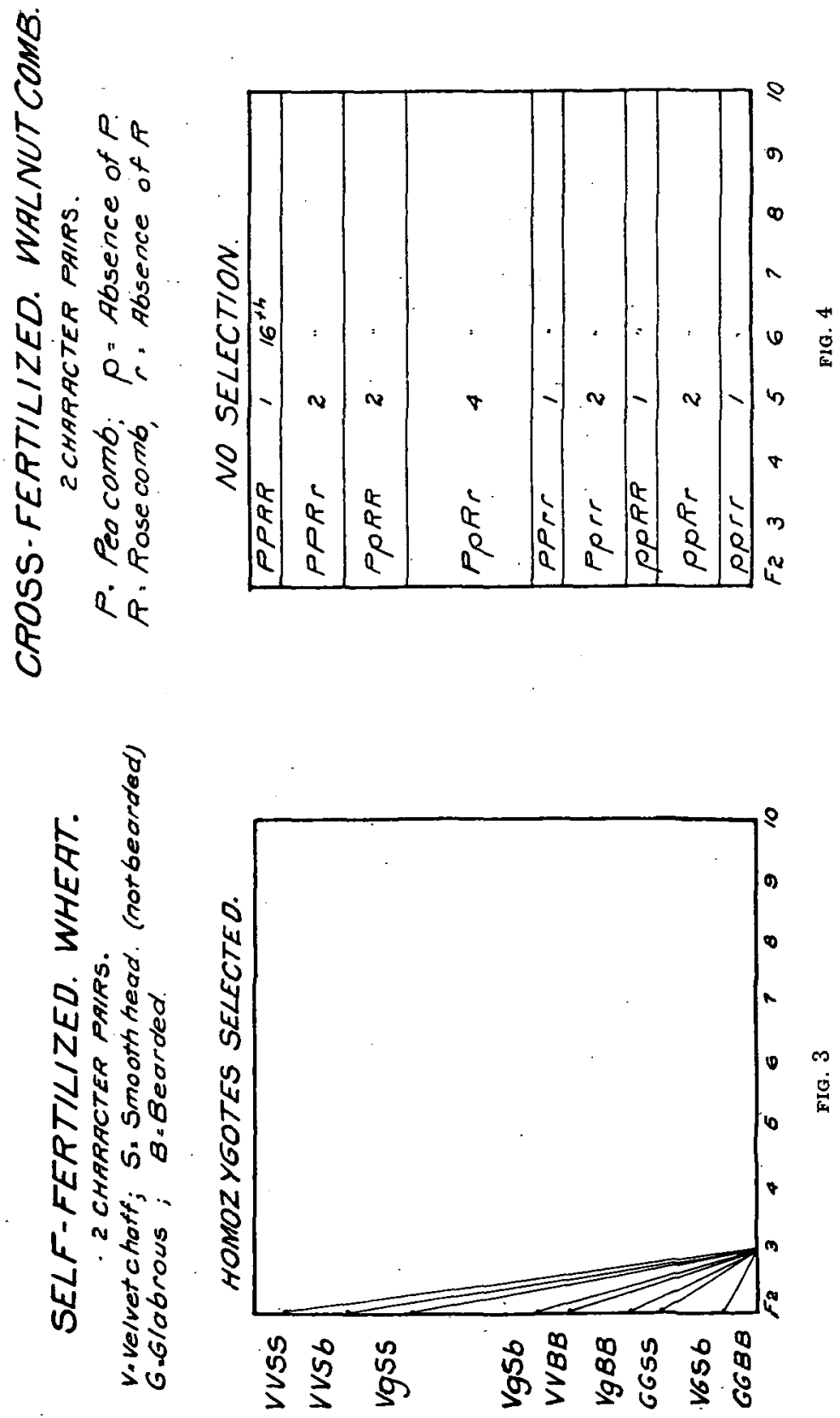
close fertilization occurs. This is shown in Fig. 4, the cross in this case being between walnut comb and plain comb fowls. The walnut comb is a compound character, one of the elements of which is the pea comb and the other the rose comb. After the cross is made, if no selection

\section{CAOSS-FERTILIZED. WALNUT COME. 2 CHARACTEA PAIA. $P=$ Pea.comb; $P=$ Absence of $P$. P. Pose; $r$. Absence of $P$.}

\section{RECESSIVES DISCAROED.}

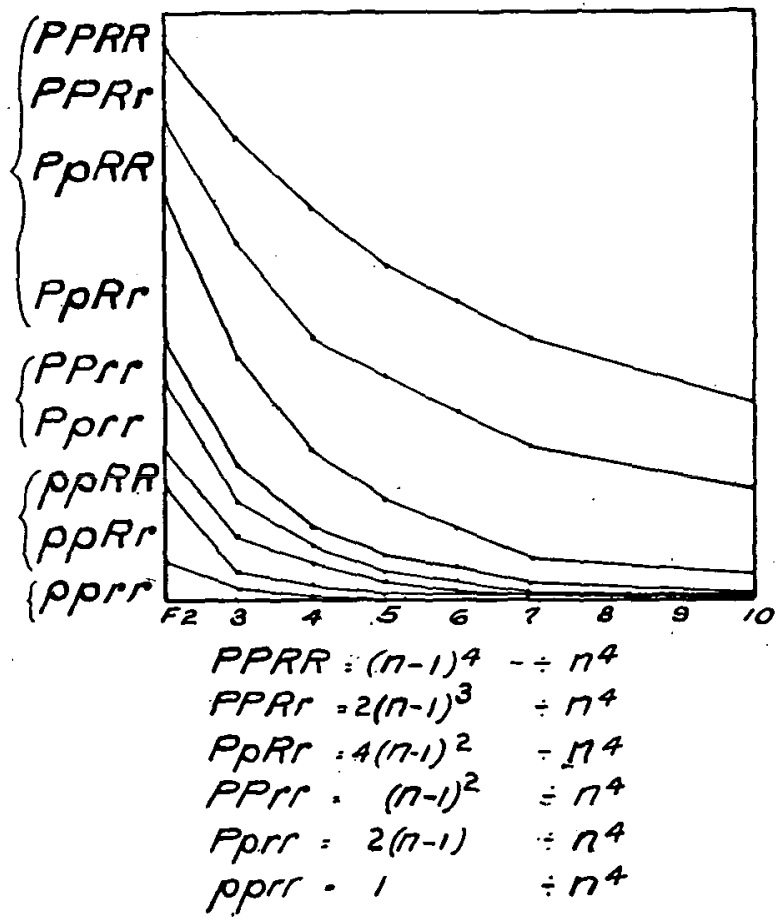

FIG. 5

with reference to comb is made and the birds are allowed to mate promiscuously, the proportion of each of the nine types present in the population remains the same indefinitely.

Fig. 5 shows what occurs in this case when all birds in the second and later generations are discarded, unless they have the walnut comb. It 
is seen that at the end of ten generations about three-eighths of the population consists of homozygote walnut comb birds. Something over oneeighth consists of birds that are homozygote with reference to the pea comb and heterozygote with reference to the rose comb, while a similar number are heterozygote for pea comb and homozygote for rose comb.

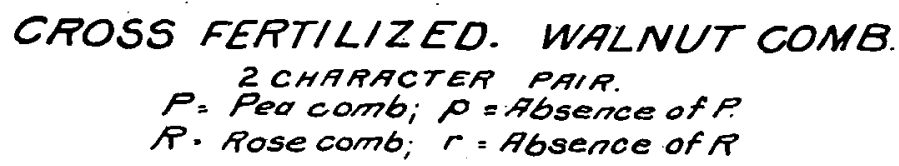

MALES TESTED.

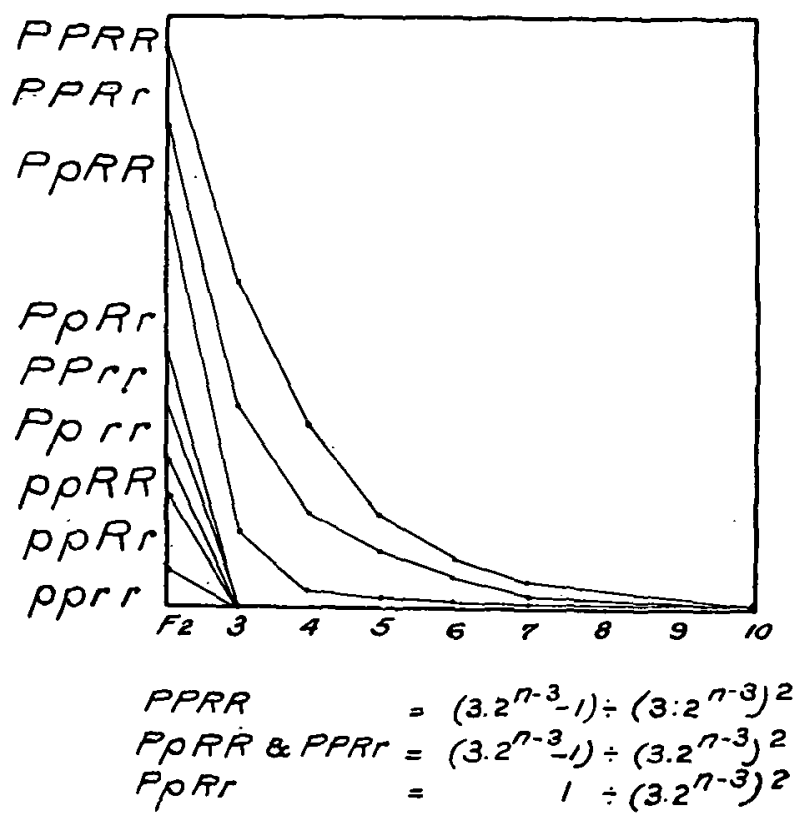

FIG. 6

A much smaller proportion of the population consists of birds that are heterozygote for both characters. The number of birds that do not have walnut combs is reduced, in ten generations, to a very small fraction of the population.

The method of selecting homozygote individuals which is applicable in the case of close fertilized plants is not always practicable with animals 

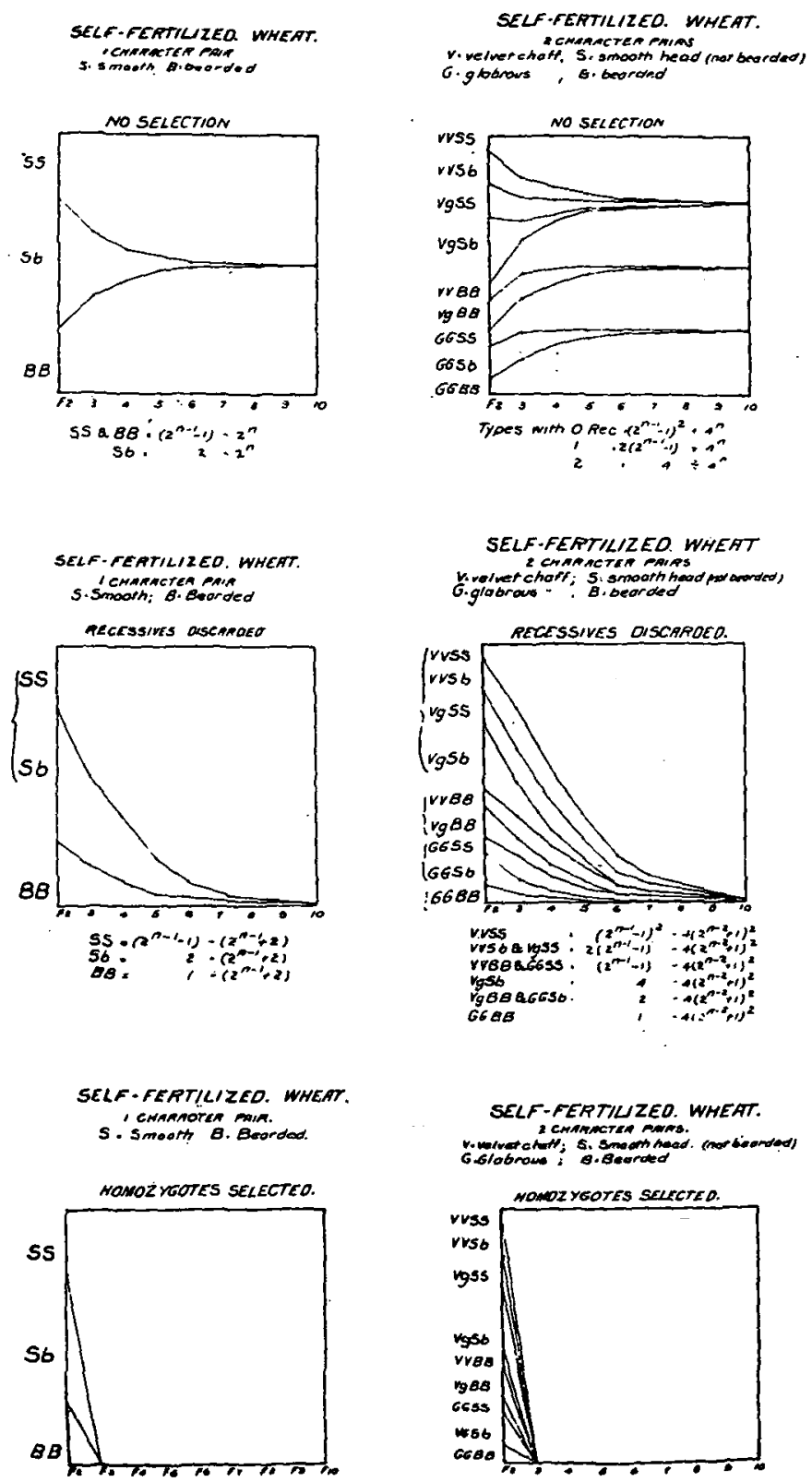


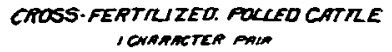

icusanctep Pato

A Poved, H- Atorned

NO SELECTION.

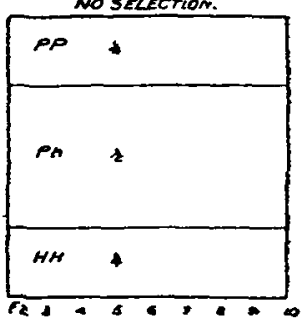

CFOSS FEFTILIZEO. WALNET COASR

2 enepactea mons.

P. Fecond. p. Absence of $F$

no seLectran

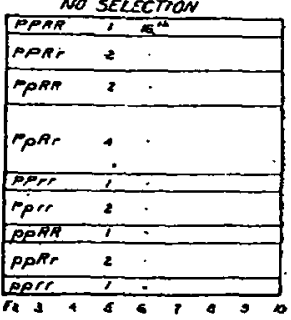

CAOSS FEATIUZEO. FULEO CATTLE. I cMandecter mar. P. Polled: Mrmarener

EROSS-FEMTHZEO. WALNUT COME.

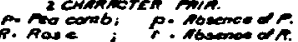

Mcesomes arscinoco.

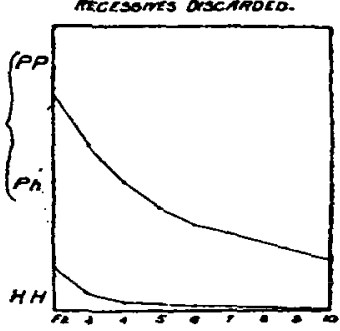

pP. $\left(n^{2}-1\right)-n^{2}$

An: $: 2(n-1)=n^{2}$

HH: $2\left(n-n^{2}-\right.$

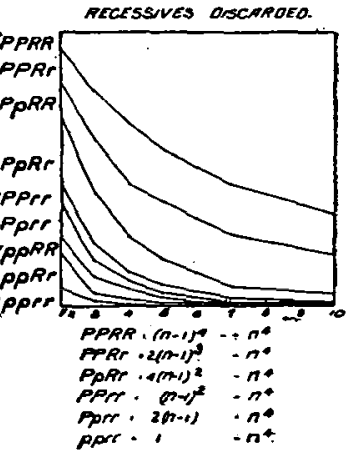

CROSS-FEATUZEO. FOLCEO CATTLE. piroved: bi Horend.

CROSS RERTILIZEO. WALNUT CONE

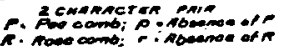
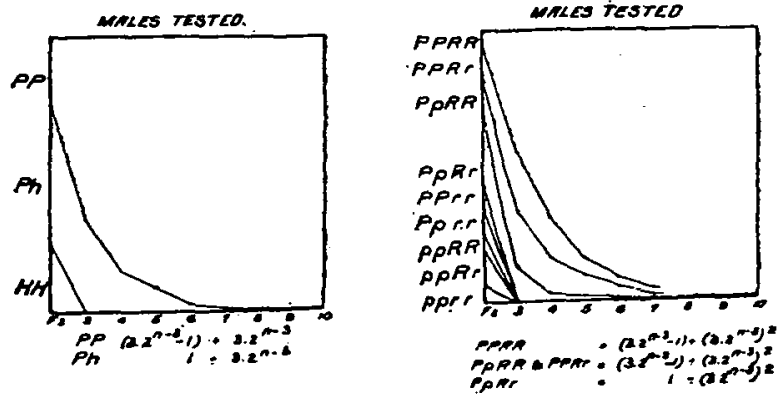
but in many cases it is practicable to select homozygote individuals of one sex. Fig. 6 shows the results that follow when all recessive females are discarded and only homozygote males are used. It is seen that after the second generation no recessives crop out, and that within ten generations the number of heterozygote individuals decreases to a very small proportion of the population.

It was Fig. 2 about which Dr. Shull, at the meeting of this association last year, stated that it illustrated the results obtained by Mr. Burbank by his method of selection, which in many cases consists of discarding undesirable forms.

It should be remembered that the principles here outlined apply only to Mendelian characters. We do not yet know whether all hereditary characters obey Mendel's law of segregation or not. If there are characters which do not obey Mendel's law then these formalæ would not apply to them.

\title{
CLONAL OR BUD VARIATION.
}

\author{
By Herbert J. Webber.
}

Contribution II, Laboratory Experimental Plant-Breeding, Cornell University.

LIMITED MEANING OF INDIVIDUALITY IN PLANTS.

Up to the present time breeders have been dealing primarily with the plant as an individual and as the unit in all considerations of variation evolution and breeding. This, however, from the standpoint of our cultivated plants is clearly an error as many of our varieties have originated as so-called bud sports or bud variations and it is clear that this type of variation must have its place in any system of plant-breeding. Animals, particularly the higher forms, are almost completely Autonomons. The animal has a definite number of legs, arms, ears, etc. It possesses almost complete bilateral symmetry. Each part is dependent on the other parts for its existence. All parts develop and grow together and die at the same time, having a more or less definite span of life. In all of the higher plants, however, there is a totally different condition. Every plant is made up of a certain number of largely independent units which may be said to be fighting with each other from birth for supremacy. Each node, internode and bud forming together a unit which is designated a phytomer is in considerable measure independent of every similar unit on the plant. To make this clear take as an illustration a tree of any sort. The first shoot produced is for a period simple and 